\title{
The Interplay of Oxidative Stress and Inflammation: Mechanistic Insights and Therapeutic Potential of Antioxidants
}

\author{
Ayman M. Mahmoud $\mathbb{D}^{1,2}$ Fiona L. Wilkinson, ${ }^{3}$ Mansur A. Sandhu, ${ }^{4}$ \\ and Adam P. Lightfoot ${ }^{3}{ }^{3}$ \\ ${ }^{1}$ Physiology Division, Zoology Department, Faculty of Science, Beni-Suef University, Egypt \\ ${ }^{2}$ Biotechnology Department, Research Institute of Medicinal and Aromatic Plants (RIMAP), Beni-Suef University, Egypt \\ ${ }^{3}$ Department of Life Sciences, Faculty of Science and Engineering, Manchester Metropolitan University, UK \\ ${ }^{4}$ Department of Biomedical Sciences, Faculty of Veterinary \& Animal Sciences, PMAS, Arid Agriculture University, Pakistan
}

Correspondence should be addressed to Ayman M. Mahmoud; ayman.mahmoud@science.bsu.edu.eg

Received 28 July 2021; Accepted 28 July 2021; Published 20 August 2021

Copyright ( 2021 Ayman M. Mahmoud et al. This is an open access article distributed under the Creative Commons Attribution License, which permits unrestricted use, distribution, and reproduction in any medium, provided the original work is properly cited.

Oxidative stress is caused by the overproduction of Reactive Oxygen Species (ROS) and diminished cellular antioxidant defenses. Under physiological conditions, ROS are involved in processes, including cellular homeostasis, modulation of cellular metabolism, signaling and redox state, and being used by the immune system to inactivate viruses and inhibit bacterial growth. However, excess ROS production can damage lipids, DNA, and proteins, which can lead to cell death. ROS-induced damage underpins various oxidative stressrelated human diseases and aging. In addition, cellular antioxidant defenses may decrease during aging, resulting in age-related increase in ROS and oxidative stress. Excess ROS can also activate proinflammatory signaling pathways and the release of multiple inflammatory mediators, such as cytokines, chemokines, eicosanoids, and others. Therefore, oxidative stress is implicated in the pathogenesis, development, and progression of a sustained inflammatory state. Furthermore, excess ROS and inflammation work together to trigger and orchestrate necrotic and apoptotic cell death, where mitochondrial dysfunction, caspase activation, and Bcl-2 family proteins are involved in ROS-mediated apoptosis. In addition, several protein kinases and signaling pathways (including mitogen-activated protein kinases, nuclear factor-kappaB, protein kinase $\mathrm{C}$, and others) modulate apoptosis depending on the cellular context.

Oxidative stress and inflammation are increasingly recognized as having key roles in the pathogenesis of various diseases, including diabetes, obesity, cancer, neurodegeneration, metabolic syndrome, cardiovascular disease, liver disease, and others. Therefore, understanding the molecular mechanisms underlying the mutual relationship between oxidative stress and inflammation can lead to the discovery of novel strategies to prevent and/or treat various diseases. Although cells are equipped with numerous nonenzymatic molecules and enzymatic scavengers of ROS, these defenses are not always adequate to attenuate the excess ROS production. Therefore, agents that can boost antioxidant defense mechanisms and prevent increased ROS generation can represent an effective treatment for oxidative stress/inflammation-related diseases.

This special issue encompasses 25 research articles, one clinical study, and 6 review articles focusing on the interplay of oxidative stress and inflammation in different disease conditions, including diabetes and its complications, liver injury, pancreatitis, lung fibrosis, ocular diseases, cardiovascular diseases, hemorrhagic shock, nonalcoholic fatty liver disease, cardiac hypertrophy, myocardial infarction, depression, celiac disease, and others.

The guest editors are pleased to present a compendium of these cutting-edge original research and review articles as follows:

Research article: "Hydrostatin-SN10 Ameliorates Pancreatitis-Induced Lung Injury by Affecting IL-6Induced JAK2/STAT3-Associated Inflammation and 
Oxidative Stress." In this article, X. Piao et al. evaluated the protective effect of the peptide hydrostatin-SN10 against lung injury associated with pancreatitis induced by taurocholate in mice. Hydrostatin-SN10 reduced malondialdehyde (MDA), proinflammatory cytokines, and the number of apoptotic cells and boosted antioxidants. These effects were associated with the suppressed IL-6-stimulated JAK2/STAT3 pathway in the lung.

Research article: "Methane-Rich Saline: A Potential Resuscitation Fluid for Hemorrhagic Shock." Based on previous studies showing the beneficial effects of methane-rich saline (MRS), used as a resuscitation fluid in animal models, Y. Tong et al. explored its protective effect in hemorrhagic shock induced in rats by bloodletting via intubation of the right femoral artery. MRS restored systemic blood pressure, ameliorated serum levels of liver and heart function markers, MDA, proinflammatory cytokines, apoptosis markers in the heart, and upregulated the PGC-1 $\alpha$ /SIRT3/SOD2 signaling pathway.

Research article: "Plant Sterol Ester of $\alpha$-Linolenic Acid Attenuates Nonalcoholic Fatty Liver Disease by Rescuing the Adaption to Endoplasmic Reticulum Stress and Enhancing Mitochondrial Biogenesis." H. Han et al. showed that nonalcoholic fatty liver disease (NAFLD) can be effectively controlled by supplementing the diet with a plant sterol ester of $\alpha$-linolenic acid as it inhibited liver steatosis, improved liver maladies, mitigated inflammatory responses, downregulated stress markers in the endoplasmic reticulum, and increased mitochondrial biogenesis.

Research article: "Flavonoids from Apios americana Medikus Leaves Protect RAW264.7 Cells against Inflammation via Inhibition of MAPKs, Akt-mTOR Pathways, and Nfr2 Activation." Q. Chu et al. investigated the anti-inflammatory activity of a flavonoid-rich Apios americana leaf extract (ALE) in vitro. Treatment with ALE reduced the release of proinflammatory cytokines, nitric oxide (NO), and oxidative stress and inhibited NF- $\kappa$ B and MAPK signaling and upregulation of HMGB1-Beclin1, Sirt1-FoxO1, and Nrf2-Keap1 pathways in lipopolysaccharide- (LPS-) induced RAW264.7 cells.

Research article: "Aronia melanocarpa Prevents AlcoholInduced Chronic Liver Injury via Regulation of Nrf2 Signaling in C57BL/6 Mice." In this study, Z. Wang et al. analyzed the components of Aronia melanocarpa (AM) and explored its effects on alcohol-induced chronic liver injury in mice. The component analysis of AM revealed 17 types of amino acids, 17 types of fatty acids, 8 types of minerals, and 3 types of nucleotides. In alcohol-induced mice, AM showed hepatoprotective effects, suppressed oxidative stress and inflammation markers, activated Nrf2 signaling, and enhanced antioxidants.

Research article: "Tetrahydroxystilbene Glucoside Ameliorates Infrasound-Induced Central Nervous System (CNS) Injury by Improving Antioxidant and Anti-Inflammatory Capacity." X. Zhou et al. studied the therapeutic effects of $2,3,5,4^{\prime}$-tetrahydroxystilbene-2-O- $\beta$-D-glycoside (THSG) in infrasound-caused injury in the CNS. THSG suppressed inflammation, oxidative stress, and apoptotic cell death, enhanced antioxidant defenses in the hippocampal tissues of the mouse model, and ameliorated the learning and memory impairments caused by infrasound.
Research article: "Comparative Study on Pulmonary Toxicity in Mice Induced by Exposure to Unflavoured and Apple- and Strawberry-Flavoured Tobacco Waterpipe Smoke." A. Nemmar et al. investigated the pulmonary toxicity induced by daily exposure to plain-, apple-flavored, and strawberry-flavored waterpipe smoking (WPS) in mice. Exposure to WPS altered lung function and morphology and induced oxidative stress and inflammation by activating NF- $\kappa$ B. The toxic effect of flavored tobacco WPS was found to be greater than that of unflavored WPS.

Research article: "Hydrogen Sulfide Protects against Paraquat-Induced Acute Liver Injury in Rats by Regulating Oxidative Stress, Mitochondrial Function, and Inflammation." In this study, Z. Liu et al. reported that exogenous hydrogen sulfide enhanced antioxidative capability, upregulated Nrf2 and SIRT3/IDH2 signaling, improved mitochondrial function, and suppressed ROS-induced NLRP3 inflammasome activation, resulting in amelioration of paraquat hepatotoxicity.

Research article: "Musa balbisiana Fruit Rich in Polyphenols Attenuates Isoproterenol-Induced Cardiac Hypertrophy in Rats via Inhibition of Inflammation and Oxidative Stress." S. Kumari et al. evaluated the protective effect of Musa balbisiana (MB) fruit pulp powder against cardiac hypertrophy induced by isoproterenol in rats and in vitro in H9c2 cells. The fruit powder ameliorated cardiac hypertrophy markers and attenuated ISO-induced inflammation and oxidative stress.

Research article: "Mesenchymal Stem Cells Attenuate Diabetic Lung Fibrosis via Adjusting Sirt3-Mediated Stress Responses in Rats." In this study, Y. Chen et al. reported the beneficial effect of mesenchymal stem cells (MSCs) in diabetes-induced lung injury. Bone marrow MSCs effectively attenuated lung fibrosis via modulating Sirt3-mediated responses, including inflammation, oxidative stress, apoptosis, autophagy, and endoplasmic reticulum stress in streptozotocin-induced diabetic rats.

Research article: "Fucoidan Ameliorates Oxidative Stress, Inflammation, DNA Damage, and Hepatorenal Injuries in Diabetic Rats Intoxicated with Aflatoxin $\mathrm{B}_{1}$." M. S. Alessia et al. indicated that the sulfated polysaccharide fucoidan can effectively normalize serum markers of liver and kidney function and attenuate inflammation, oxidative stress, and DNA damage in STZ-diabetic rats.

Research article: "Lactobacillus delbrueckii Ameliorates Intestinal Integrity and Antioxidant Ability in Weaned Piglets after a Lipopolysaccharide Challenge." In this study, F. Chen et al. reported that dietary supplementation of Lactobacillus delbrueckii improved intestinal integrity and immune response and attenuated intestinal oxidative damage in LPS-challenged piglets.

Research article: "Water-Soluble Pristine $\mathrm{C}_{60}$ Fullerenes Inhibit Liver Fibrotic Alteration and Prevent Liver Cirrhosis in Rats." H. Kuznietsova et al. evaluated the impact of the effective free radical scavenger, pristine $C_{60}$ fullerenes $\left(\mathrm{C}_{60} \mathrm{FAS}\right)$, on liver fibrosis and cirrhosis and their ability to modulate the main growth factor receptors involved in liver fibrogenesis. $\mathrm{C}_{60} \mathrm{FAS}$ attenuated hepatocyte injury, oxidative stress, and connective tissue deposition in $\mathrm{N}$ - 
diethylnitrosamine/carbon tetrachloride-intoxicated rats. The computational modeling revealed that $\mathrm{C}_{60} \mathrm{FAS}$ can block the hinge prohibiting ATP binding to EGFR and FGFR.

Research article: "Vagus Nerve Stimulation Ameliorates Renal Ischemia-Reperfusion Injury through Inhibiting NF$\kappa \mathrm{B}$ Activation and iNOS Protein Expression." M. Wang et al. found that vagus nerve stimulation treatment attenuated renal ischemia/reperfusion injury in rats by inhibiting iNOS expression, oxidative stress, and inflammation via $\mathrm{NF}-\kappa \mathrm{B}$ inactivation.

Research article: (E)-N ${ }^{\prime}$-(1-(7-Hydroxy-2-Oxo-2HChromen-3-Yl) Ethylidene) Benzohydrazide, a Novel Synthesized Coumarin, Ameliorates Isoproterenol-Induced Myocardial Infarction in Rats through Attenuating Oxidative Stress, Inflammation, and Apoptosis.” A. Feriani et al. investigated the ameliorative effect of a novel synthesized coumarin, 7-hyd.HC, on isoproterenol-induced myocardial infarction in rats. 7-hyd.HC ameliorated serum cardiac function markers, normalized cardiac function and ECG pattern, and mitigated oxidative stress, apoptosis, and fibrinogen production in ISO-induced rats.

Research article: "Nauclea latifolia Sm. Leaf Extracts Extenuates Free Radicals, Inflammation, and DiabetesLinked Enzymes." F. N. Iheagwam et al. evaluated the in vitro anti-inflammatory, antioxidant, $\alpha$-amylase, and $\alpha$ glucosidase inhibitory activities of ethanolic and aqueous extracts of Nauclea latifolia leaves. Both extracts showed positive effects and could serve as antioxidant and antiinflammatory candidates.

Research article: " $\beta$-Phenethyl Isothiocyanate Induces Cell Death in Human Osteosarcoma through Altering Iron Metabolism, Disturbing the Redox Balance, and Activating the MAPK Signaling Pathway." H. Lv et al. reported that $\beta$ phenethyl isothiocyanate (PEITC) reduced cell viability, inhibited proliferation, and caused $\mathrm{G}_{2} / \mathrm{M}$ cell cycle arrest in MNNG/HOS, U-2 OS, MG-63, and 143B cell lines and delayed tumor growth in a xenograft osteosarcoma mouse model. The chemopreventive effect of PEITC was associated with the induction of oxidative stress and triggering ferroptosis, apoptosis, and autophagy.

Research article: "Tanshinone IIA Protects against Acute Pancreatitis in Mice by Inhibiting Oxidative Stress via the Nrf2/ROS Pathway." In this investigation, W. Chen et al. reported that tanshinone IIA, the active constituent of Salvia miltiorrhiza Bunge, conferred protection against acute pancreatitis in mice by suppressing oxidative stress via activation of the Nrf2/ROS pathway.

Research article: "Oxidant/Antioxidant Profile in the Thoracic Aneurysm of Patients with the Loeys-Dietz Syndrome." M. E. Soto et al. reported that redox homeostasis is altered in thoracic aortic aneurysms (TAA) in Loeys-Dietz syndrome patients, favoring ROS overproduction that contributes to the decline of antioxidant defenses.

Research article: "Chitoheptaose Promotes Heart Rehabilitation in a Rat Myocarditis Model by Improving Antioxidant, Anti-Inflammatory, and Antiapoptotic Properties.” Q. Zhao et al. emphasized the cardioprotective role of chitosan oligosaccharides (COS) in LPS-stimulated RAW264.7 cells and a rat model of myocarditis. Seven COS were evaluated for their reactive scavenging, inflammatory and apoptotic factors, and myocardial protective effects. Chitoheptaose was the most effective among the tested COS where it showed a myriad of cardioprotective roles in the myocarditis model via its antioxidant, anti-inflammatory, and antiapoptotic activities.

Research article: "Mulberry Fruit Prevents Diabetes and Diabetic Dementia by Regulation of Blood Glucose through Upregulation of Antioxidative Activities and CREB/BDNF Pathway in Alloxan-Induced Diabetic Mice." A. Y. Min et al. investigated the effect of the ethyl acetate fraction of the ethanolic extract of mulberry fruit (MFE) on biochemical and behavioral deficits in alloxan-induced diabetic mice. The results showed that MFE exerts antidiabetic and neuroprotective effects by upregulating antioxidative activities and the $\mathrm{p}-\mathrm{CREB} / \mathrm{BDNF}$ pathway.

Research article: "FNDC5 Attenuates Oxidative Stress and NLRP3 Inflammasome Activation in Vascular Smooth Muscle Cells via Activating the AMPK-SIRT1 Signal Pathway." In this study, B. Zhou et al. evaluated the protective role of fibronectin type III domain-containing 5 (FNDC5) in angiotensin II- (Ang II-) induced vascular oxidative stress and inflammation. The results revealed that FNDC5 deficiency aggravates while exogenous FNDC5 alleviates the Ang II-induced vascular oxidative stress and NLRP3 inflammasome activation via the AMPK-SIRT1 signal pathway in vascular smooth muscle cells.

Research article: "Inhibition of P2X7 Purinergic Receptor Ameliorates Cardiac Fibrosis by Suppressing NLRP3/IL-1 $\beta$ Pathway." J. Zhou et al. examined the involvement of P2X7 purinergic receptor (P2X7R) in the development of cardiac fibrosis using a transverse aortic constriction (TAC) mouse model and cardiac fibroblasts (CFs) hyperstimulated by TGF- $\beta 1$ for $48 \mathrm{~h}$. P2X7R activation promoted cardiac fibrosis, and its inhibition protected against CF activation and cardiac fibrosis by modulating the NLRP3/IL-1 $\beta$ pathway.

Research article: "Inhibition of JNK Alleviates Chronic Hypoperfusion-Related Ischemia Induces Oxidative Stress and Brain Degeneration via Nrf2/HO-1 and NF- $\kappa$ B Signaling." M. S. Khan et al. aimed to determine redox imbalance and c-Jun N-terminal kinase-associated detrimental effects in chronic ischemia and the inhibitory effect of a specific JNK inhibitor (SP600125) on JNK-mediated brain degeneration in adult mice. The results indicated that the inhibition of JNK reduced neurodegeneration and regulated cognitive dysfunction in the chronic ischemic mouse model.

Research article: "PARK7 Diminishes Oxidative StressInduced Mucosal Damage in Celiac Disease." A. VeresSzékely et al. investigated the role of increased Parkinson's disease 7 (PARK7) levels on the epithelial cell and mucosal integrity of the small intestine. PARK7 induced the expression of stress-response elements, and PARK7-binding Comp23 reduced the oxidative damage of duodenal epithelial cells by upregulating Nrf2- and P53-regulated genes.

Clinical study: "The Beneficial Effects of Alpha Lipoic Acid Supplementation on Lp-PLA2 Mass and Its Distribution between HDL and apoB-Containing Lipoproteins in Type 2 Diabetic Patients: A Randomized, Double-Blind, 
Placebo-Controlled Trial." N. Baziar et al. aimed to investigate the effect of alpha-lipoic acid (ALA) on the lipoprotein-associated phospholipase A2 (Lp-PLA2) mass and its distribution in diabetic patients. They concluded that ALA may decrease the cardiovascular risk in type 2 diabetic patients by reducing the oxidized low-density lipoprotein (ox-LDL) and Lp-PLA2 mass and improving the Lp-PLA2 distribution among lipoproteins in type 2 diabetic patients.

Review article: "Unveiling the Role of Inflammation and Oxidative Stress on Age-Related Cardiovascular Diseases." In this review, A. J. P. Oliveira de Almeida et al. discussed the basics of inflammation and oxidative stress, including the crosstalk between them, and the implications on agerelated cardiovascular diseases. The authors recommended therapies counteracting oxidative stress and inflammation without inhibiting their physiological functions as promising targets in the search for healthy aging.

Review article: "PGC-1 $\alpha$, Inflammation, and Oxidative Stress: an Integrative View in Metabolism." S. Rius-Pérez et al. highlighted the role and pathways of peroxisome proliferator-activated receptor- $\gamma$ coactivator- (PGC-) $1 \alpha$ as a transcriptional coactivator. The review introduced a summary of the role of PGC- $1 \alpha$ acting as an interesting therapeutic target and an essential node connecting metabolic regulation, redox control, and inflammatory pathways.

Review article: “Targeting Inflammatory-Mitochondrial Response in Major Depression: Current Evidence and Further Challenges." A. P. V. Visentin et al. provided a review of major depressive disorder and its association with inflammation, mitochondrial dysfunction, and oxidative stress and discussed the putative links between these events. They highlighted the need of new investigations focusing on the antidepressant effects of antioxidants.

Review article: "Coumarins as Modulators of the Keap1/Nrf2/ARE Signaling Pathway.” E. H. M. Hassanein et al. provided a review compiling the research findings of seventeen coumarin derivatives of plant origin as antioxidant and anti-inflammatory agents, emphasizing the role of Nrf2 activation in their pharmacological activities. They utilized molecular docking simulations to investigate the potential binding mode of these coumarins with Keap1 as a strategy to disrupt Keap1/Nrf2 protein-protein interaction and activate Nrf2 signaling.

Review article: "Adjuvant Therapies in Diabetic Retinopathy as an Early Approach to Delay Its Progression: The Importance of Oxidative Stress and Inflammation." In this review, R. R. Robles-Rivera et al. focused on the promising values of nutraceuticals and different compounds as an adjuvant approach in the treatment of diabetic retinopathy to diminish its progression.

Review article: "Potential Protective and Therapeutic Roles of the Nrf2 Pathway in Ocular Diseases: An Update." Here, M. Wang et al. focused on the possible involvement of Nrf2, a redox-sensitive transcription factor, in the occurrence and development of ocular diseases. They have also discussed the role of noncoding RNAs and exogenous compounds, which control the expression of Nrf2 through different pathways, in ocular diseases.
The editors anticipate this special issue to be of interest to the readers and expect researchers to benefit from making further progress in understanding the mechanisms of oxidative stress and inflammation crosstalk in different diseases and the therapeutic potential of antioxidants.

\section{Conflicts of Interest}

The editors declare that they have no conflicts of interest regarding the publication of this special issue.

\section{Acknowledgments}

We would like to thank the authors for submitting their insightful and interesting research for publication and the reviewers for sharing their expertise, constructive critiques, and their contributions to improve the manuscripts.

Ayman M. Mahmoud Fiona L. Wilkinson Mansur A. Sandhu Adam P. Lightfoot 